\title{
QUALCOM : une expérience sur la qualification des comportements d'une lampe robotique
}

Joffrey Becker, Virginie André and Alain Dutech

\section{OpenEdition \\ Journals}

\section{Electronic version}

URL: https://journals.openedition.org/tc/10771

DOI: 10.4000/tc. 10771

ISSN: 1952-420X

Publisher

Éditions de l'EHESS

\section{Electronic reference}

Joffrey Becker, Virginie André and Alain Dutech, "aualcom : une expérience sur la qualification des

comportements d'une lampe robotique", Techniques \& Culture [Online], Varia, Online since 11 February 2019, connection on 29 September 2022. URL: http://journals.openedition.org/tc/10771 ; DOI: https:// doi.org/10.4000/tc. 10771

This text was automatically generated on 29 September 2022

All rights reserved 


\title{
QUALCOM : une expérience sur la qualification des comportements d'une lampe robotique
}

\author{
Joffrey Becker, Virginie André and Alain Dutech
}

\begin{abstract}
« Dans cette expérience, il vous est demandé de décrire les animations qui vous seront présentées par cette lampe par le biais du questionnaire qui vous a été remis. Pour ce faire, vous utiliserez un ou plusieurs termes simples selon ce qu'évoque pour vous chaque animation. Il vous sera également demandé d'évaluer chaque animation en lui attribuant des valeurs numériques allant de 1 à 9 . Si l'animation que vous observez n'évoque rien pour vous, cochez la case correspondante sur le questionnaire sans chercher à lui trouver un terme correspondant et sans lui attribuer de valeur numérique. Vous pouvez choisir jusqu'à trois termes si vous souhaitez être plus précis dans votre description ou si les mouvements de la lampe vous évoquent des choses différentes. N'hésitez pas à demander à la personne qui vous accompagne de rejouer une animation si vous le jugez nécessaire. De même, n'hésitez pas à employer les termes qui vous viennent spontanément à l'esprit. Le but de cette expérience n'est pas de vous faire trouver des réponses exactes au questionnaire que vous allez remplir, mais plutôt d'avoir une idée de ce que les mouvements de la lampe vous inspirent. Vous êtes ainsi complètement libres d'utiliser les termes qui vous semblent les mieux correspondre à ce que vous avez vu. À l'issue de cette première étape, un rapide entretien enregistré sera conduit avec vous à propos des termes que vous avez choisis. »
\end{abstract}




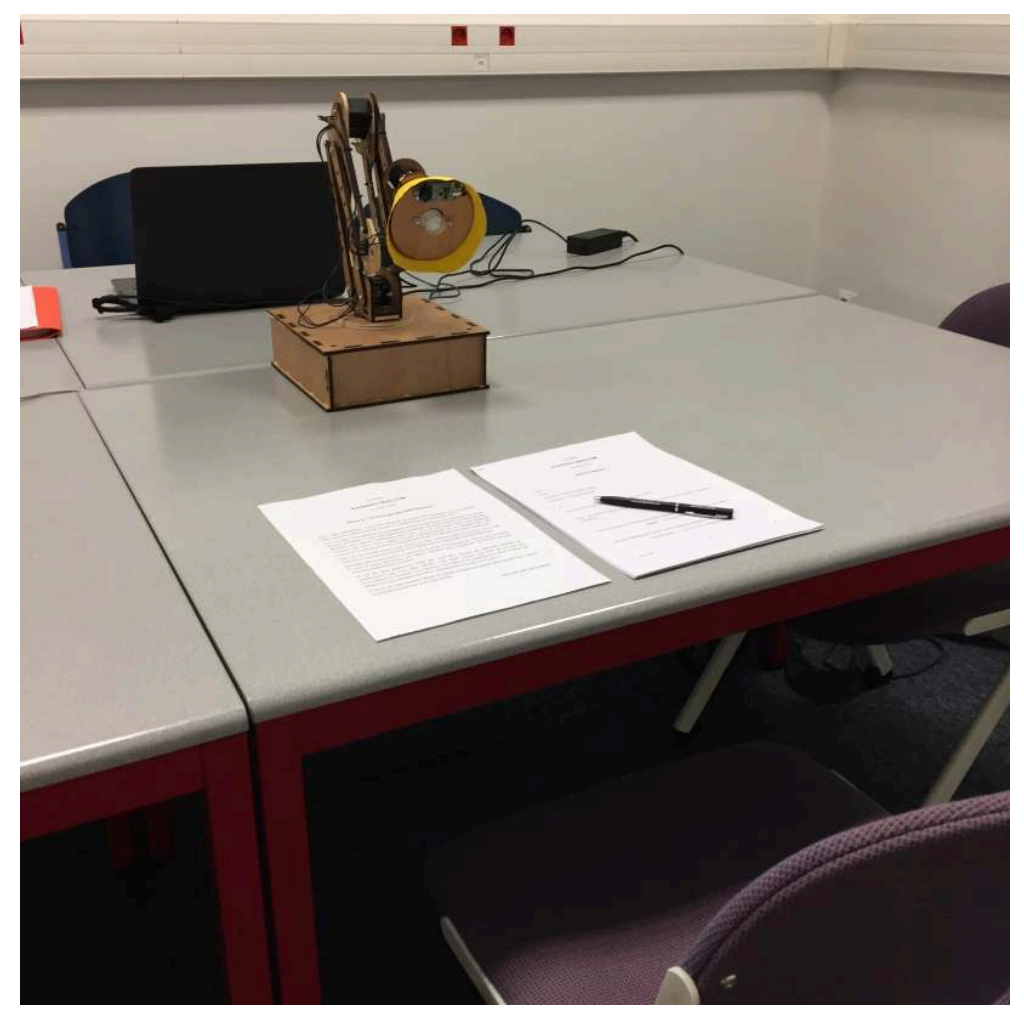

1 : CONfIgURATION MATÉRIELLE dE L'EXPÉRIENCE Qualcom.

CLICHÉ : J. BECKER, 2016.

1 En entrant dans l'espace réservé à l'expérience (fig. 1) qui va nous servir d'appui dans cet article, chaque participant est invité à s'assoir et à lire cette consigne. Face à lui, une lampe de bois motorisée est sur le point de s'animer. Cette lampe est au cœur de plusieurs expériences conduites par un groupe de chercheurs issus de disciplines comme la robotique, l'intelligence artificielle, les neurosciences, la philosophie, la linguistique et l'anthropologie, et dont le but est de mieux saisir les processus cognitifs à l'œuvre lors des interactions avec les robots dits sociaux.

2 La robotique est le domaine privilégié des automatismes industriels et il peut sembler au premier abord malaisé de penser les robots à travers les intentions propres qu'on leur prête ou d'imaginer à quel degré nous trouvons qu'ils nous ressemblent. Les systèmes robotiques sont en passe de profondément changer nos sociétés. Que l'on songe simplement à la manière dont ils agissent dans bien des domaines des activités humaines, sur les réseaux, dans les transports ou au cœur de l'activité de travail. Réfléchir à l'impact des technologies issues de la robotique est un exercice d'anticipation difficile. Il implique finalement assez peu qu'on s'intéresse à la façon dont on les considère ou au type de relation que l'on peut entretenir directement avec elles.

3 Or les robots sont fréquemment pensés par analogie avec des corps vivants, et notamment humains (Vidal 2007 ; Becker 2015). L'apparition récente de la robotique dite sociale et de son lot d'humanoïdes, comme l'essor de l'intelligence artificielle, montre très clairement comment une pensée de l'activité des machines se développe par référence directe à celles des corps. Ces développements laissent supposer quels types de lien nous pourrions entretenir avec les machines, de quelle façon la ressemblance au corps doit favoriser l'existence d'un espace partagé au sein duquel une 
sorte de confiance (préalable à une forme renouvelée de coopération avec les machines) peut paraître possible. Mais on doit moins y voir un phénomène résultant des avancées récentes des sciences et des techniques de l'ingénieur qu'un renforcement des fondations du domaine de la robotique et des rapports que l'on entretient depuis longtemps avec les créatures artificielles.

4 L'ambiguïté soulevée par les machines anthropomorphes, comme la façon dont celles-ci nous poussent à nous interroger sur la pertinence des représentations mécaniques de la nature, n'est pas un phénomène proprement lié aux développements récents de la robotique sociale. Elle traverse la philosophie grecque de l'action dès le Ive siècle avant notre ère. L'automate y forme l'occasion d'une confusion entre la mécanique et la vie (Espinas 1903) qui trouvera de nombreux prolongements chez Borelli, Leibniz ou Descartes, puis dans la confection des automates de l'âge classique par Jacques Vaucanson, Pierre et Henri-Louis Jaquet-Droz, mais aussi Johann Wolfgang von Kempelen. Ces machines, en empruntant à la fois à la forme et à l'activité du corps, suscitent une curiosité certaine parmi leurs spectateurs. Elles créent des espaces de spéculation autour des principes qui les animent (Riskin $2003: 627$ ).

La robotique qui est pratiquée actuellement dans les laboratoires s'inscrit dans la continuité de ces travaux. Comme avec les automates de l'âge classique, les fonctions vitales restent une source d'inspiration permettant, par effet de fragmentation et de rétroaction, de penser la vie et notamment ce que nous sommes (Becker 2012a). Mais cette inspiration dépasse le seul domaine des sciences et des techniques. À cette référence au vivant que ces dernières empruntent, on doit ajouter l'ensemble très vaste des références propres à chaque personne ayant à interagir avec ces dispositifs. La perception des actions de telles machines implique une autonomie prêtée qui s'ajoute à leur autonomie réelle (Becker 2015). Si la robotique imite ainsi la vie en fabriquant des objets animés, elle l'imite également à partir de l'étude des processus d'interaction entre les êtres; elle cherche en quelque sorte à saisir ce qui constituerait l'algorithme de la vie sociale (Becker 2012b). Qu'une machine épouse la forme d'un corps humain ou qu'elle reste abstraite, qu'elle soit pourvue de langage ou qu'elle reste muette, qu'elle se mette en mouvement ou qu'elle s'arrête sans raison apparente, une « personne » nous semble présente dès que nous avons affaire à un robot. Comment comprendre cette attitude paradoxale qui fait du robot une "personne » dont la nature machinique est pourtant vérifiée à travers l'expérience? Devenons-nous animistes au contact d'une machine qui nous ressemble? Que se passerait-il si les machines n'empruntaient pas, dans leur forme comme dans leurs mouvements, aux actions humaines?

6 L'objet du présent article est, en s'appuyant sur la description d'une expérience, de montrer à quel point la confrontation avec de telles machines reste marquée par l'incompréhension. Lorsque nous sommes face à ces dispositifs, le sens que l'on peut prêter à leurs mouvements ne nous semble en réalité jamais vraiment clair. Les phénomènes de personnification qui semblent à l'œuvre dès que nous sommes en leur compagnie sont peut-être plus complexes qu'il n'y parait.

7 Nous défendrons, ici, l'hypothèse que les robots sont des objets techniques dont l'animation ne favorise pas l'apparition d'une pensée anthropomorphique à proprement parler, mais rend surtout visible la mise en œuvre d'une pensée fondée de manière bien plus large sur l'analogie. En d'autres termes, la confrontation avec la lampe robotisée qui nous servira de point de départ n'engage pas simplement les humains dans une sorte de repli anthropomorphique inné, à partir duquel il leur 
semble possible de donner du sens à ce qu'ils perçoivent. Nous verrons plutôt que le corps humain ne sert pas de référence première dès qu'il s'agit d'interpréter le « comportement » d'un robot.

8 Après avoir présenté le contexte de l'expérience et les méthodes employées pour la réaliser, nous détaillerons les résultats qu'elle a permis d'obtenir. Ces derniers, qui sont issus de l'étude des espaces sémantiques occupés lors d'un exercice de qualification des mouvements de la lampe robotisée, nous permettront de souligner à la fois le caractère contingent et extrêmement flottant des relations entretenues avec des artefacts dotés d'une vie. Ils nous montreront comment le sens que l'on prête à l'activité de tels objets s'appuie sur des éléments connus, certes directement dépendants de l'expérience des individus, mais qui dépassent de loin un seul rapport de forme avec l'humain.

\section{Au-delà de la forme humaine}

Les anthropologues travaillant sur la robotique ont très vite remarqué que les processus encadrant l'activité des robots ressemblent beaucoup à ceux qui président plus généralement à l'apparition d'artefacts dotés de subjectivité (Vidal 2007; Becker 2015). Comme nombre de ces objets, l'existence des robots dépend principalement de deux facteurs. D'une part, ils sont issus d'une procédure complexe de fragmentation et d'assemblage qui implique un ensemble de savoirs spécialisés. Et d'autre part, ils sont voués à l'interaction sociale en mettant en jeu différents niveaux d'animation et en étant par conséquent dotés d'une autonomie dont ils ne disposent pas forcément. L'étude de ces processus croise en fait deux approches distinctes. Elle vise d'abord à saisir les principales étapes de la fabrication de ces objets. Et elle nécessite également qu'on s'intéresse aux cadres dans lesquels les interactions avec eux ont d'ordinaire cours.

9 L'approche que nous privilégierons ici suivra ces deux modalités de la conception des robots et de leur autonomie. Elle mettra par conséquent l'accent sur les techniques employées pour concevoir un dispositif technique, mais également sur l'étude des espaces sémantiques dans lesquels se situent les qualificatifs choisis par des « interactants » humains pour évaluer ses mouvements. Cette approche nous permettra de mieux comprendre comment une machine peut être qualifiée de sociale en s'appuyant pour cela sur un dispositif expérimental pensé initialement pour résister aux projections anthropomorphiques.

10 En effet, une des principales difficultés lorsqu'on travaille avec les outils de la robotique dite sociale est que le rapport envers la forme humaine est au cœur de la plupart des études consacrées aux robots. Cette approche anthropocentrée relève en réalité d'une perspective qui porte l'intérêt sur les dynamiques d'attachement envers les machines anthropomorphes (ou plus largement biomorphes) sans toutefois replacer ces machines dans le paysage plus vaste de la robotique où elles restent, malgré la visibilité qui leur est accordée, encore largement minoritaires. La littérature produite en sciences humaines et sociales traite ainsi la question de l'anthropomorphisme en robotique en considérant des catégories de phénomènes hétérogènes qui pourtant trouvent à chaque fois leur source dans l'imitation du corps humain.

11 Dans leur conception autant que dans les effets qu'ils produisent, les robots anthropomorphes tissent de drôles de liens avec l'humain. Ces simulations d'êtres vivants placent par exemple sur un même plan les sujets et les objets "possédables " 
(Richardson 2016). Ils constituent autant d'écrans sur lesquels sont projetés nos fantasmes (Giard 2017 : 118). Ils forment un lieu où se réinvente la nature (Helmreich 1998) et le corps (Suchman 2007). Ce sont également, en raison de leur incapacité à saisir la complexité des relations sociales ou à faire sens du monde qui les entoure, autant de pièges anthropomorphiques dans lesquels nous serions disposés à tomber (Vidal 2017 ; Tisseron 2015).

12 L'activité de telles machines engage celles et ceux qui les observent dans un jeu consistant à la fois à faire l'hypothèse des qualités sociales du robot tout en cherchant à en saisir l'intériorité. Les roboticiens travaillant sur la cognition de l'interaction homme-robot et la robotique sociale l'ont bien compris (Siciliano \& Khatib 2016). L'étude des modèles mentaux que les humains ont des robots, des théories cognitives sur l'interaction, de l'expression de (pseudo)-émotions et de la reconnaissance des activités humaines leur servent avant tout à faciliter les relations qui se construisent entre les humains et les machines. En s'appuyant sur le constat que les interaction hommes-machines suivent les règles des interactions sociales entres les humains (Reeves \& Nass 1996), l'empathie devient un enjeu majeur de ces recherches qui conduisent «naturellement» à des robots dont la forme, le comportement et les processus invitent leurs utilisateurs à reconnaître de l'humain en eux.

13 Ces robots, on le voit, sont avant tout considérés pour la ressemblance qu'on leur prête, les inférences qu'ils font naître ou l'espace commun qui progressivement s'invente autour d'eux. Certes, la présence robotique, comme l'autonomie qui lui est associée, suscite tout autant des sentiments mêlés de fascination, de confusion, d'étrangeté et de malaise ${ }^{1}$. Mais cette présence est en réalité plus complexe qu'il n'y paraît. Emmanuel Grimaud et Zaven Paré ont bien montré que les présences associées à l'activité du robot Geminoïd, qui combinent celle du professeur Ishiguro et des chercheurs de son équipe, celle de son manipulateur et enfin celle du système autonome lui-même, conduisent dans le cours même de l'interaction à un exercice de requalification; les mouvements du Geminoïd l'apparentant à une créature semi-vivante. Cette distinction que les humanoïdes nous invitent à faire entre ce qui relève de la vie et ce qui n'en relève pas, a également fait l'objet d'une étude récente conduite par Denis Vidal. Pour lui, la question de l'anthropomorphisme prend un tour nouveau si on la considère à travers les multiples façons dont les sociétés jouent avec les catégories qui leur servent à ordonner le monde. Denis Vidal montre que le principal enjeu de la rencontre avec un robot consiste en une mesure de son autonomie et ses limites ${ }^{2}$. Cette mesure vise à vérifier, pour le dire autrement, que la machine en est bien une. Pour autant, une fois la nature machinique du robot confirmée, cela n'empêche pas les humains de se comporter avec lui comme s'il s'agissait d'une " personne » (Vidal 2017).

Comment comprendre cette attitude paradoxale? En traçant des continuités entre les intériorités des humains et des machines tout en constatant qu'une discontinuité se maintient, notamment au niveau de l'apparence, les interactions entre les robots et les humains ne révèlent-ils pas qu'une forme renouvelée d'animisme est à l'œuvre? Philippe Descola a bien établi que l'animisme implique un tel rapport de continuité et de discontinuité (Descola 2005). Dans l'archipel des sociétés animistes, si l'ensemble des existants non-humains est doté d'une intériorité identique à celle des humains, c'est principalement sur la base d'une différenciation morphologique que vont s'établir les rapports d'identité et d'altérité. On pressent que les relations avec les robots relèvent de rapports tout à fait comparables. Or, rien n'est en réalité moins sûr. 
15 Dans les mondes de l'animisme, on ne projette pas seulement les attributs d'une personne, mais également la sociabilité propre qui s'établit entre les membres d'une même espèce, d'espèces différentes, ou entre les êtres vivants et les artefacts (Viveiros de Castro 2014; Kohn 2017 ; Santos-Granero 2009). Le statut de «personne» qui est inféré lors d'un échange avec un robot semble, lui, tout autant marqué par l'instabilité que par l'incertitude. Il semble ressortir d'une identité aussi complexe que cumulative, l'ontologie d'une machine faisant l'objet de transformations diverses dans le temps même de l'interaction (Becker 2015). Par ailleurs, et bien que les machines semblent avoir un monde perceptif qui leur est propre et dont on peut borner les frontières, les expériences conduites dans le domaine des interactions humains-robots montrent la chose suivante : attribuer la qualité de sujet à une machine n'implique pas pour autant qu'on infère à propos des relations sociales qui pourraient prévaloir dans une société de robots, comme cela serait le cas dans le type d'inférence ontologique animiste.

16 L'activité de ces machines reste ainsi toujours marquée par une somptueuse opacité (Latour 2010 : 22). Et c'est cette opacité qu'il nous semble important d'aborder ici. Indépendamment de sa forme ou des principes qui l'animent, une machine est toujours une boite noire dont les actions seules semblent permettre l'ouverture. Ces objets, en raison des connaissances très partielles que nous avons de leur mécanisme interne, agissent sur nous comme de véritables catalyseurs de croyance. Or les mécanismes perceptifs impliqués lors des rencontres entre les humains et les robots restent, comme l'ont bien remarqué les commissaires de l'exposition Persona (Grimaud \& Taylor 2016) encore mal connus. Il est ainsi important de se demander « qui est là ? » lors d'une telle rencontre; de "s'interroger sur les paramètres qui favorisent la perception d'une présence dans une situation donnée, et qui font que cette présence est ramenée ou non à des familles connues d'humains, d'animaux, d'esprits, de divinité, de fantômes, etc. " (Grimaud et al. $2016: 13$ ).

17 Si l'on peut ainsi constater la centralité toujours accordée à la figure humaine dans l'étude des modalités de l'interaction entre humains et machines, il est désormais temps de chercher à y inclure des machines qui échappent aux travers de la ressemblance avec le corps afin de voir comment l'activité de ces dernières est agissante sur nous et de quelle manière. Il s'agira ici de porter l'intérêt sur un objet dont la forme cherche à s'extraire des références au vivant et de prêter plus particulièrement attention au potentiel qu'ont ces objets à nous engager sur la voie des inférences (Grimaud \& Becker 2019). Cette démarche nous conduira à montrer comment l'animation d'un artefact est issue d'un processus technique dont l'effet est d'être interprété en mobilisant des catégories employées non seulement pour décrire l'activité des objets mais aussi celle d'êtres vivants d'une assez grande variété. Elle s'inscrit en cela dans la perspective d'une anthropologie des imbrications croisées entre les processus vitaux et techniques (Pitrou 2016). On verra, à partir d'une expérience que nous allons à présent nous appliquer à décrire, que les analogies employées pour décrire les mouvements d'un objet issu du quotidien relèvent de catégories diverses et que l'intériorité potentielle qu'elles exposent ressort d'un entrelacement complexe allant au-delà de l'imputation systématique d'un caractère humain envers les objets. 


\section{La lampe du groupe PsyPhiNe}

Cette approche tente en fait de regrouper différents intérêts relatifs à l'ancrage disciplinaire des auteurs et de manière plus large ceux du groupe de recherche dans lequel ils inscrivent leurs travaux. Il serait difficile de résumer les questionnements et les préoccupations extrêmement divers qui animent les débats du groupe PsyPhiNe. Comme il serait difficile, ici, d'envisager avec quelle difficulté cette équipe tente de conduire un travail résolument interdisciplinaire. Pour ce qui concerne les auteurs du présent article, l'apport d'une approche expérimentale réside dans les implications qu'a la robotique sur leurs disciplines elles-mêmes. Par exemple, du point de vue de la socio-linguiste Virginie André, l'intérêt d'expérimenter à partir d'une lampe robotisée repose notamment sur la façon dont cette dernière met à l'épreuve les modèles servant à décrire les interactions, verbales ou non, entre pairs. Les critères qui servent d'appui à la recherche dans un tel domaine résistent-ils dès lors que l'interaction implique un humain et une machine? Du point de vue du roboticien Alain Dutech, un tel cadre expérimental a pour vocation de mieux saisir les mécanismes qui permettent l'interaction et par conséquent d'améliorer les dispositifs techniques à vocation sociale. Enfin, du point de vue de l'anthropologue Joffrey Becker, l'approche expérimentale a le double intérêt de constituer un cadre d'observation participante privilégié pour qui s'intéresse à la mise en œuvre de tels dispositifs, et elle offre aussi une occasion rare d'envisager les diverses façons dont les humains considèrent les robots; comment ces derniers sont perçus ; comment et selon quels critères ils parviennent, ou échouent, à s'insérer dans une dynamique sociale plus large.

Né à Nancy en 2011 des échanges entre une dizaine de chercheurs en intelligence artificielle, en psychologie, en philosophie, en neurosciences et en sociolinguistique autour de la cognition naturelle et artificielle, le groupe PsyPhiNe s'est engagé dans l'élaboration d'une série d'expériences permettant de mieux saisir les dynamiques d'attribution fréquemment observables lors des interactions entre humains et machines, ou de manière plus étendue lors des rapports interspécifiques. Arrivée plus récemment au sein du groupe, l'anthropologie y trouve aujourd'hui sa place. La présence d'un anthropologue au sein du groupe est la conséquence d'un premier séjour de deux mois de J. Becker au Laboratoire Lorrain de Recherche en Informatique et ses Applications pendant sa thèse de doctorat en 2011, puis d'échanges répétés avec les roboticiens du groupe au cours des années suivantes. Ces échanges ont abouti, grâce au soutien de la Maison de Sciences de l'Homme, de l'Université et de la Région Lorraine, à l'organisation d'un séjour d'études postdoctorales d'un an, lors duquel il était demandé à l'anthropologue d'aider le groupe dans la conception des expériences en s'appuyant sur la lampe robotisée et de les réaliser.

De manière générale, les expériences conduites par le groupe PsyPhiNe visent à neutraliser la forme humaine à laquelle la plupart des travaux de recherches en robotique dite sociale font explicitement ou implicitement référence, lorsqu'elles s'appuient notamment sur le langage ou sur l'utilisation d'un registre de symboles partagés par les « interactants » en situation expérimentale. Que se passe-t-il lorsqu'on tente d'enlever, dans la forme comme dans les actes, toute référence symbolique aux comportements humains? Prêtera-t-on tout aussi facilement que nous semblons le faire lorsque nous sommes confrontés à quelque chose qui nous ressemble, une intériorité, des sentiments et pourquoi pas de la conscience à un objet, ainsi que le 
suggèrent les travaux menés dès le milieu des années 1940 par Fritz Heider et Marianne Simmel ? Pour tenter d'apporter un début de réponse à ces questions, les expériences conduites par le groupe PsyPhiNe s'appuient sur la mise en présence de participants humains et d'un objet animé, une lampe robotisée créée au printemps 2015, inspirée à la fois par le projet Pinokio d'Adam Ben-Dror et Shanshan Zhou ${ }^{3}$, et du court métrage d'animation Luxo Jr réalisé en 1986 par John Lasseter pour la société de production américaine Pixar Animation Studios ${ }^{4}$.

La lampe utilisée pour les expériences du groupe PsyPhiNe est faite de contreplaqué léger dont les pièces ont été découpées au laser puis assemblées autour de cinq moteurs Robotis Dynamixel AX-12 et AX-18. L'abat-jour de la lampe consiste en un morceau de carton jaune et abrite une ampoule centrale ainsi qu'une petite caméra installée juste au-dessus de l'ampoule. Ces deux derniers éléments ne sont pas utilisés au cours de l'expérience qui va être décrite plus loin. Le caractère "artisanal» de cet objet lui donne un caractère singulier qui, malgré sa forme immédiatement reconnaissable, la démarque des objets manufacturés produits en série. Les moteurs de la lampe actionnent cinq liaisons pivot, offrant ainsi cinq degrés de liberté, en rotation. En partant de la base de la lampe, on trouve successivement les cinq articulations suivantes (fig. 2) :

- rotation de la lampe sur sa base selon un axe vertical. Cette rotation offre une amplitude de 270 degrés, $\boldsymbol{\theta}_{5} \in[-135,135]$

- rotation du premier bras de la lampe dans un plan vertical, cela permet globalement d'avancer ou d'éloigner la lampe. Amplitude : $\boldsymbol{\theta}_{4} \in[50,100]$

- rotation du deuxième bras de la lampe dans un plan vertical, cela permet globalement de monter ou descendre l'abat-jour. Amplitude : $\boldsymbol{\theta}_{3} \in[-10,80]$

- rotation de l'abat-jour dans un plan vertical, cela permet d'incliner l'abat-jour vers le bas ou le haut. Amplitude : $\boldsymbol{\theta}_{2} \in[0,50]$

- rotation de l'abat-jour autour d'un axe vertical, cela permet une rotation à droite ou à gauche. Amplitude $: \boldsymbol{\theta}_{1} \in[-90,90]$ 

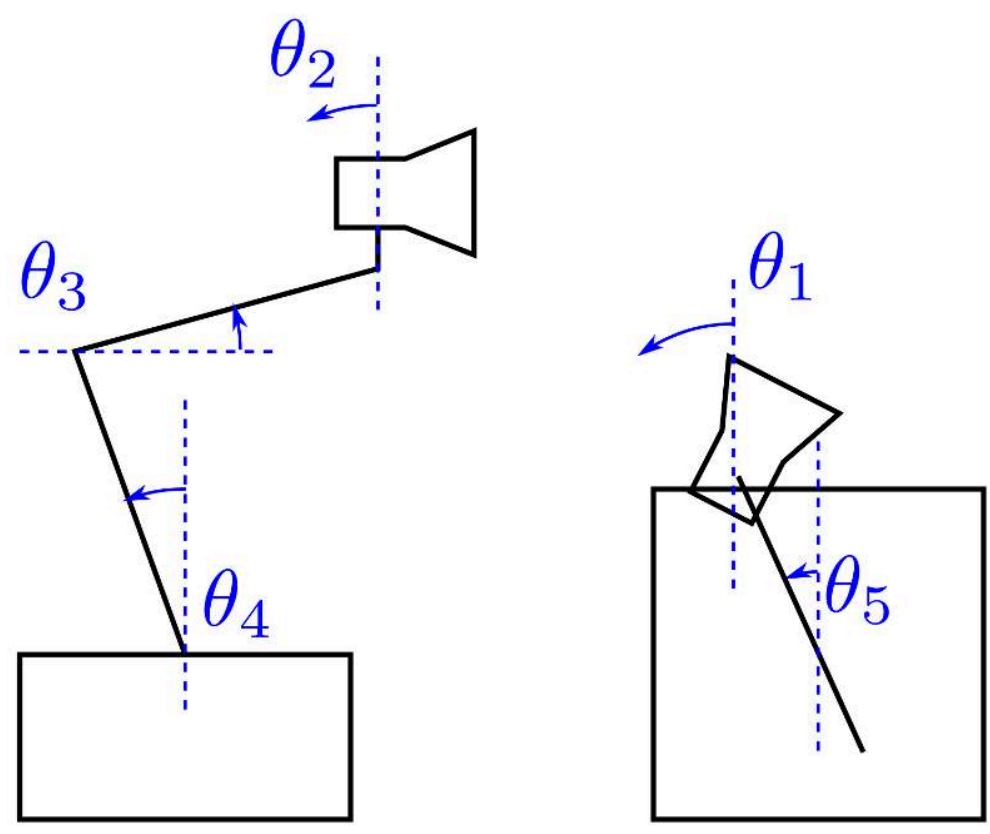

\section{2 : DESCRIPTION DES DEgRÉS DE LIBERTÉ DE LA LAMPE.}

Comme les bras de la lampe sont constitués de deux segments articulés, celle-ci est assimilable à un robot parallèle car plusieurs chaînes cinématiques indépendantes relient la base de la lampe à son abat-jour. Cette architecture en simplifie la manipulation. Par exemple, changer l'angle de rotation $\boldsymbol{\theta}_{4}$ du premier bras n'a pas d'influence sur le bras suivant. L'angle $\boldsymbol{\theta}_{3}$ entre le deuxième bras et l'horizontale n'est pas changé. En revanche, cela complique un peu le calcul de la cinématique inverse de la lampe qui ne peut être assimilée à une simple chaîne cinématique.

Ce robot parallèle est commandé par un ordinateur portable auquel les moteurs sont raccordés sur un port USB. Dans le cadre de l'expérience qui nous intéressera ici, une interface de commande a été créée. Celle-ci a été développée en langage python et s'appuie sur la bibliothèque logicielle pypot pour communiquer avec les moteurs et TkInter pour l'interface graphique. L'interface de commande permet de connaître, à tout moment, l'état des moteurs (angle de rotation, température), de mettre ou non les moteurs en tension et de faire exécuter différents mouvements au robot. Nous avons choisi de contrôler les moteurs en position, c'est à dire qu'une commande atomique $\mathrm{u}=\left(\boldsymbol{\theta}, d_{t}\right)$ envoyée à un moteur contient l'angle de rotation $\boldsymbol{\theta}$ que doit atteindre ce moteur et le temps $d t$ qu'il doit mettre pour atteindre cette position. Un mouvement consiste ainsi en une séquence de positions cibles pour les cinq moteurs, auxquelles s'ajoute le temps séparant chacune de ces poses. Les mouvements sont en conséquence assez saccadés puisque la vitesse de déplacement du robot s'annule à chaque position intermédiaire avant d'augmenter à nouveau pour aller rejoindre la position suivante. Il est cependant assez simple de créer un comportement. Il suffit en effet d'enregistrer une succession de poses pour la lampe et d'indiquer ensuite les intervalles de temps qui les séparent. 


\section{L'expérience QUALCOM}

C'est selon ce principe d'animation ${ }^{5}$ que la lampe du groupe PsyPhiNe a été utilisée lors d'une expérience conduite au printemps 2016, lors de laquelle il était demandé à chaque participant de construire une structure grâce à des petites briques de bois afin de faire en sorte que la lampe s'allume. La lampe avait été dotée pour cette expérience de mouvements de type émotionnel et manifestait de la joie, de l'ennui, de la curiosité, etc. Autant de fragments de comportements qui devaient faciliter l'interaction des participants humains avec elle et les engager progressivement dans la voie des inférences. Mais ces mouvements, rapidement réalisés par des étudiants et ne prenant en compte que des critères d'évaluation esthétique très généraux, n'avaient pu être testés. Le sens qu'ils pouvaient prendre lors de l'interaction avec un humain restait en conséquence difficilement évaluable. C'est pour cette raison que l'expérience QUALCOM a été mise en œuvre, afin de vérifier si les mouvements initialement conçus sont bien porteurs d'un même sens pour les personnes qui les perçoivent. Son principal objectif est ainsi de déterminer dans quels termes un groupe d'humains décrit et qualifie les comportements qui leurs sont présentés. Le but de cette expérience n'est pas d'évaluer la qualité des animations jouées par la lampe afin qu'ils soient porteurs d'un même sens pour tout le monde, mais plutôt de voir dans quelle mesure les comportements déjà produits par cet objet génèrent ou non des interprétations partagées.

On peut facilement imaginer combien il est difficile de transposer à une lampe des mouvements relevant d'expressions émotionnelles humaines. Comment des mouvements comme la joie, le désespoir, la vigilance, la peur, la désapprobation, la curiosité pour le jeu ou pour le joueur, l'ennui ou la contrariété peuvent faire sens dès lors qu'ils sont donnés à voir par une lampe robotique? Pour essayer d'apporter une réponse à cette question, dix comportements potentiellement non-signifiants ont d'abord été ajoutés à la liste de mouvements existants. Ces mouvements ont été créés en faisant varier des paramètres d'amplitude, d'intensité, de durée et de vitesse et ont été insérés en alternance avec les mouvements de la liste existante. Ils ne répondent en revanche à aucun critère esthétique. Certains de ces mouvements dessinent des formes géométriques, d'autres consistent en des contorsions erratiques et incohérentes. C'est à partir de cette nouvelle liste de dix-neuf mouvements qu'a lieu l'expérience. Ces mouvements sont désignés soit par un nom lorsqu'ils ont été conçus initialement pour véhiculer une émotion, soit par un numéro pour les mouvements a priori neutres. Précisons qu'au moment où ils leurs sont montrés, les participants n'en connaissent pas les intitulés: JOIE, DIX, DÉSESPOIR, DEUX, VIGILANCE, NEUF, CURIOSITÉ JOUEUR, TROIS, PEUR, HUIT, DÉSAPPROBATION, QUATRE, CURIOSITÉ JEU, SEPT, ENNUI, CINQ, CONTRARIÉTÉ, UN, SIX.

Installé face à la lampe robotisée, et accompagné par un chercheur, chaque participant est invité à reporter sur un questionnaire un, deux ou trois termes servant à décrire les animations jouées par l'objet. La lampe montre successivement des comportements qui, selon nous, correspondent à des émotions ou ne sont porteurs d'aucun sens particulier. Il est bien précisé aux participants que la lampe est contrôlée par le chercheur. Pour chaque comportement, il est demandé au participant de qualifier par écrit ce qui vient d'être perçu. Chaque participant peut ainsi choisir entre un et trois mots clés. Il lui est également demandé d'attribuer, pour chaque animation jouée par la lampe, des valeurs (selon un axe positif/négatif, et un axe éveil-léthargie). Ces valeurs et les descriptions 
correspondantes doivent permettre de voir si un consensus se dégage sur chacun des mouvements avec pour idée d'éventuellement pouvoir replacer chaque comportement dans un graphe circumplex élaboré à partir des travaux de Barrett et Russell (1998). Si le participant hésite, il peut demander au chercheur de rejouer l'animation autant de fois que souhaité. Enfin, si un mouvement joué par la lampe ne lui évoque rien, le participant peut cocher l'option correspondante dans le questionnaire mis à sa disposition. On passe alors au mouvement suivant.

À l'issue de cette étape, il est demandé à chacun, lors d'un court entretien enregistré, de donner des détails sur les termes qui ont été employés dans la description. Cette dernière étape permet d'accéder de manière plus précise aux raisons qui ont motivé chez chacun le choix de tel ou tel terme. Il est espéré, à partir de la description que font les participants, de voir une cohérence dans la façon dont les comportements émotionnels de la lampe sont perçus. On espère également que ces descriptions nous donneront une meilleure idée de ce qui est perçu lors d'une interaction plus longue avec la lampe.

L'expérience QUALCOM s'est tenue tout au long du mois de novembre 2016 sur plusieurs sites ; au Laboratoire Lorrain de Recherche en Informatique et ses Applications, sur le Campus Lettres et Sciences Humaines de l'Université de Lorraine, dans un bar de la vieille ville de Nancy, et au domicile de certains participants. Il s'agit d'une expérience en mobilité dont l'objectif a été de rassembler une population assez variée, issue de milieux sociaux différents. Vingt-quatre personnes se sont portées volontaires pour y participer, treize femmes et onze hommes, âgés de 12 à 65 ans et dont les niveaux d'études varient entre le collège et le doctorat.

\section{Les participants face à la lampe}

L'inventaire des termes employés pour décrire l'activité de la lampe révèle d'emblée toute la complexité qui émane de la confrontation avec ses mouvements (fig. 3). Il laisse au premier abord le sentiment que ces derniers véhiculent peu de sens. On peut noter qu'indépendamment des mouvements montrés, deux grandes catégories émergent. D'une part, les participants usent du registre des expressions émotionnelles pour décrire les mouvements qu'ils perçoivent. Si la surprise, la peur ou la curiosité sont les termes les plus fréquemment employés, la lampe leur apparaît également comme timide, elle interroge, se méfie, s'étonne. D'autre part, les participants dotent la lampe de capacités de perception. Celle-ci leur apparaît non pas comme un objet qui projette de la lumière (ce qui est attendu d'un tel objet mais contredit dès qu'il commence à bouger), mais plutôt comme quelque chose qui surveille, qui regarde, qui scanne, qui explore son environnement. Il est ainsi assez surprenant que dans un contexte d'expérimentation où il est dès le début précisé qu'il ne s'agit aucunement d'une situation d'interaction - il est aussi clairement mentionné que la lampe est sous le contrôle du chercheur -, une sensibilité spécifique semble pourtant se manifester aux participants. Mais il y a peut-être plus. Car la lampe, à travers ses mouvements, a la capacité d'évoquer bien d'autres choses que des capacités à percevoir ou à éprouver. Elle évoque parfois un animal, un sujet dansant ou faisant du sport et, en quelques occasions, un objet doté d'une toute autre fonction (comme une pelleteuse). 


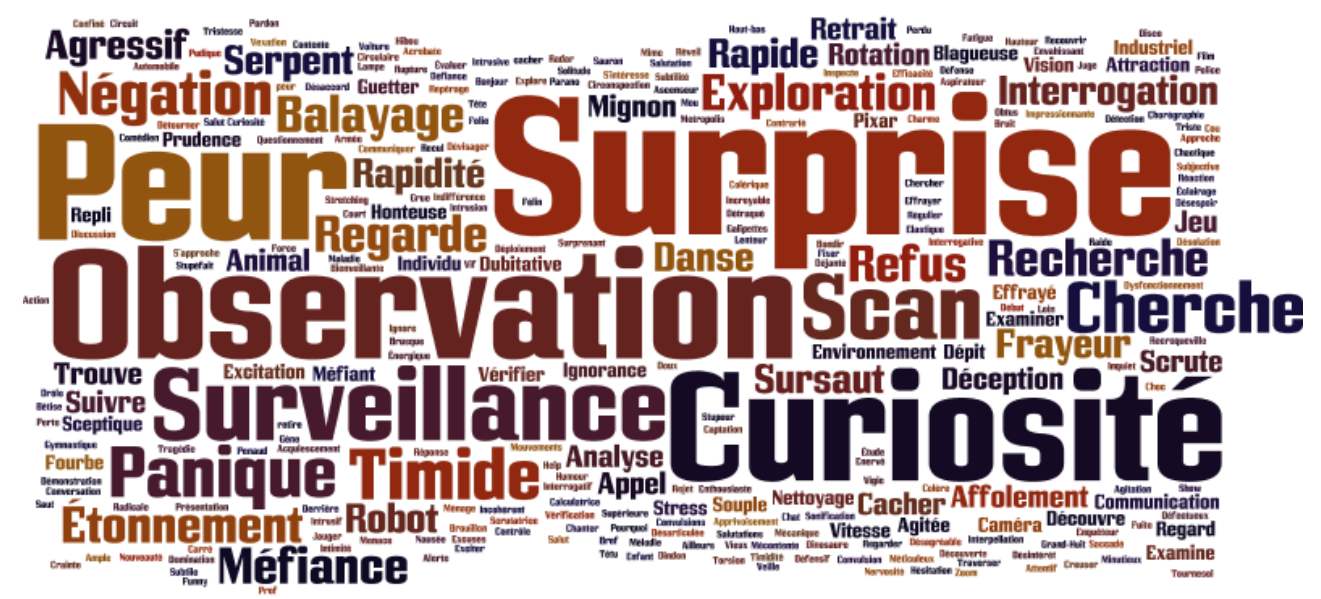

3 : LISTE DES MOTS EMPLOYÉS PAR LES PARTICIPANTS POUR QUALIfIER LES ANIMATIONS MONTRÉES PAR LA LAMPE. LES TERMES LES PLUS gROS DANS L'IMAgE SONT CEUX EMPLOYÉS LE PLUS SOUVENT.

L'analyse des espaces sémantiques occupés par les différents qualificatifs choisis pour décrire l'activité de la lampe confirme ce foisonnement des évocations. Elle montre également que les mouvements initialement pensés pour faire sens ne sont pas toujours bien compris par les participants. Ainsi, si la PEUR fait presque consensus en étant décrite avec le terme " peur » ou avec des termes du champ sémantique de la peur par 22 personnes sur 24 , les autres mouvements ne bénéficient pas systématiquement de la même unanimité. Trois comportements sont par exemple décrits avec les mêmes termes ou avec des termes appartenant au même champ sémantique par plus de la moitié des participants : par 17 (DÉSAPPROBATION), 15 (VIGILANCE) et 14 (CURIOSITÉ JEU) personnes. Trois comportements sont décrits avec les mêmes termes ou avec des termes appartenant au même champ sémantique par moins de la moitié des participants : 11 (CURIOSITÉ JOUEUR), 8 (CONTRARIÉTÉ) et 7 (DÉSESPOIR) personnes. Un comportement signifiant pour nous (ENNUI) ne l'est pas pour les participants, soit il est qualifié tout à fait autrement, soit il n'évoque rien aux participants (9 non réponses). Un comportement est qualifié par le champ lexical des antonymes de la signification que nous lui avions attribuée (la JOIE est qualifiée par le champ lexical de la colère et de la peur par 9 personnes). Un comportement que nous avions qualifié de non signifiant (10 personnes) est unanimement qualifié par le champ lexical de l'observation par les participants. Les autres comportements non-signifiants pour nous sont très différemment qualifiés ou non qualifiés par les participants.

Les entretiens enregistrés permettent d'aller plus loin dans l'étude des espaces sémantiques. Ils permettent notamment de mieux saisir les identifications induites par le mouvement de la lampe. L'objet est par exemple considéré pour autre chose que luimême en étant assimilé avec plus ou moins de détails à un corps humain ou animal en acte. Certains participants vont ainsi identifier la partie haute de la lampe à une tête ${ }^{6}$. Certains assimilent également la lampe à un animal : ce qui m'est vraiment venu c'est l'image de l'autruche / là il y avait un côté euh très marmotte je trouvais / ça me fait penser aux animaux qui s- qui se dressent / j'ai mis aussi animal euh recroquevillé et court / la façon dont il 
bougeait enfin faisait penser à un dinosaure. Cette identification prend également la forme d'une analogie avec une action accomplie dans un autre contexte ou qui renvoie à des capacités d'agir propres aux humains ou aux animaux : rechercher par terre un objet / examiner sous toutes les coutures / obtus têtu / danse / il recule comme quand on voit un truc qui peut nous surprendre / prof et colère (...) je voyais l'enseignant euh tu vas faire ton travail (...) qui montrait du doigt / enfants galipettes / j'ai ma fille qui commence à faire des mouvements un peu dans ce genre-là / comme un gamin qu'on aurait pris la main dans le sac / comme si d'un coup elle se rendait compte qu'elle avait oublié quelque chose / j'avais l'impression qu'elle lisait ce qu'il y avait sur ma feuille. Certains participants vont également créer une situation autour des mouvements de la lampe : enfin moi ce que j'imaginais c'est ça ce que ça m'évoquait c'était ça c'était de la découverte par exemple dans un environnement inconnu / on a l'impression qu'elle a perdu un truc / elle a fait une bêtise / on dirait un gamin qui fait le cochon pendu en fait / elle regarde le lever de soleil (...) j'ai imaginé que c'était la planète du Petit Prince et que le soleil se levait suffisamment souvent / c'est surveillance du contexte / comme quand on est dans une cachette qu'on sort la tête on regarde à droite on regarde à gauche avant de se lancer / il a l'air de se débattre contre une petite chose qui l'attaquait. Les participants attribuent aussi à la lampe des émotions ou des sentiments de manière directe: honteuse / curieuse / timide / elle n'était pas contente / humour / innocence / espiègle. Ils la comparent parfois à des personnages réels ou fictifs : la Reine d'Angleterre / salut de comédien / Sauron / enfant turbulent. Certains participants identifient enfin la lampe à un partenaire communicatif : mettons que je lui accorde euh je sais pas un aspect un peu humain ou un échange le début d'un échange j'associe le fait qu'on commence pas un échange comme ça / je le trouvais un peu agité pour un début de conversation / le fait de se détourner de l'interlocuteur de regarder sur le côté c'est j'ai mis méfiance / refus de parler.

29 L'objet peut aussi être considéré par identification à un autre objet : j'ai eu l'impression d'être face à un scanner / ascenseur rapide / je dirais plus il (...) je le v-je le vois pas comme une lampe en fait vu que ça a pas été allumé / une boule / lampe Pixar / un phare. En quelques occasions la lampe robotisée en reste une : c'est quand même une machine / robot / robot cassé et vieux. Enfin ses mouvements sont parfois perçus à travers un cadre relationnel élaboré par les participants eux-mêmes : j'ai mis qu'elle n'en avait rien à faire de moi / j'ai mis s'intéresse à ce que j'ai dans les mains / c'est comme si j'étais pas là / on se découvre quoi / j'ai mis présentation salutation là j'ai peut-être euh là j'ai peut-être un peu trop extrapolé dans le sens euh interaction euh / envie de communiquer chanter / je trouve ça assez envahissant dqu'elle s'approche aussi près de de moi.

Si l'on doit noter que certains participants expriment une difficulté à verbaliser ce qu'ils perçoivent, et que d'autres éprouvent quelques scrupules à personnifier la lampe (c'est vraiment bizarre de dire la lampe regarde mais bon), la façon dont elle est nommée, elle aussi, varie. Le pronom « elle » est certes le plus fréquemment employé. Toutefois, certains participants généralisent en utilisant "on ». Cette généralisation est justifiée par le fait que c'est généralement ce qu'on fait (sous-entendu en tant qu'humain) lorsque l'on fait ce type de mouvement (souvent avec sa tête) : on regarde de tous les côtés / ce que je m'imaginais au moment là c'était une scène où il y a quelque chose qui a l'air inoffensif mais on est pas sûr quoi / on regarde. Le pronom « il » est aussi employé, dans de rares cas en alternance avec «elle »: qu'il serait désolé qu'il s'excuserait / il se met un peu en-il se lève comme ça plusieurs fois je crois / on a l'impression qu'il veut paraître plus grand / l'impression qu'il découvre / il remonte comme ça / plutôt il comme un robot quoi. 
Si l'étude des qualificatifs montre que l'activité de la lampe robotisée implique une identité aussi complexe que vague, les valeurs que nous avons demandées aux participants d'attribuer pour chaque mouvement ne montrent rien en particulier (fig. 4). Il était initialement attendu de cette évaluation qu'elle nous aide à mieux comprendre comment des consensus pouvaient se former autour de chaque mouvement, et à voir si ces derniers étaient considérés comme des expressions de type émotionnel. Or, bien que le registre émotionnel soit fréquemment employé pour décrire l'activité de la lampe, les émotions considérées par les participants semblent avant tout dépendre de ce qu'ils ont en tête au moment où la lampe se met en mouvement. Ainsi, un même mouvement pourra paraitre plutôt positif pour certains, négatif pour d'autres ou même neutre. Le mouvement de PEUR par exemple, qui a pourtant été quasiment unanimement reconnu comme tel, oscillera ainsi, selon les participants, entre une bonne ou une mauvaise forme de surprise. De même, lorsqu'on leur demande d'évaluer l'énergie d'un mouvement, certains participants vont considérer que la lampe est énergique puisqu'elle bouge, tandis que d'autres vont attribuer une valeur en fonction de la vitesse avec laquelle l'objet joue son animation.

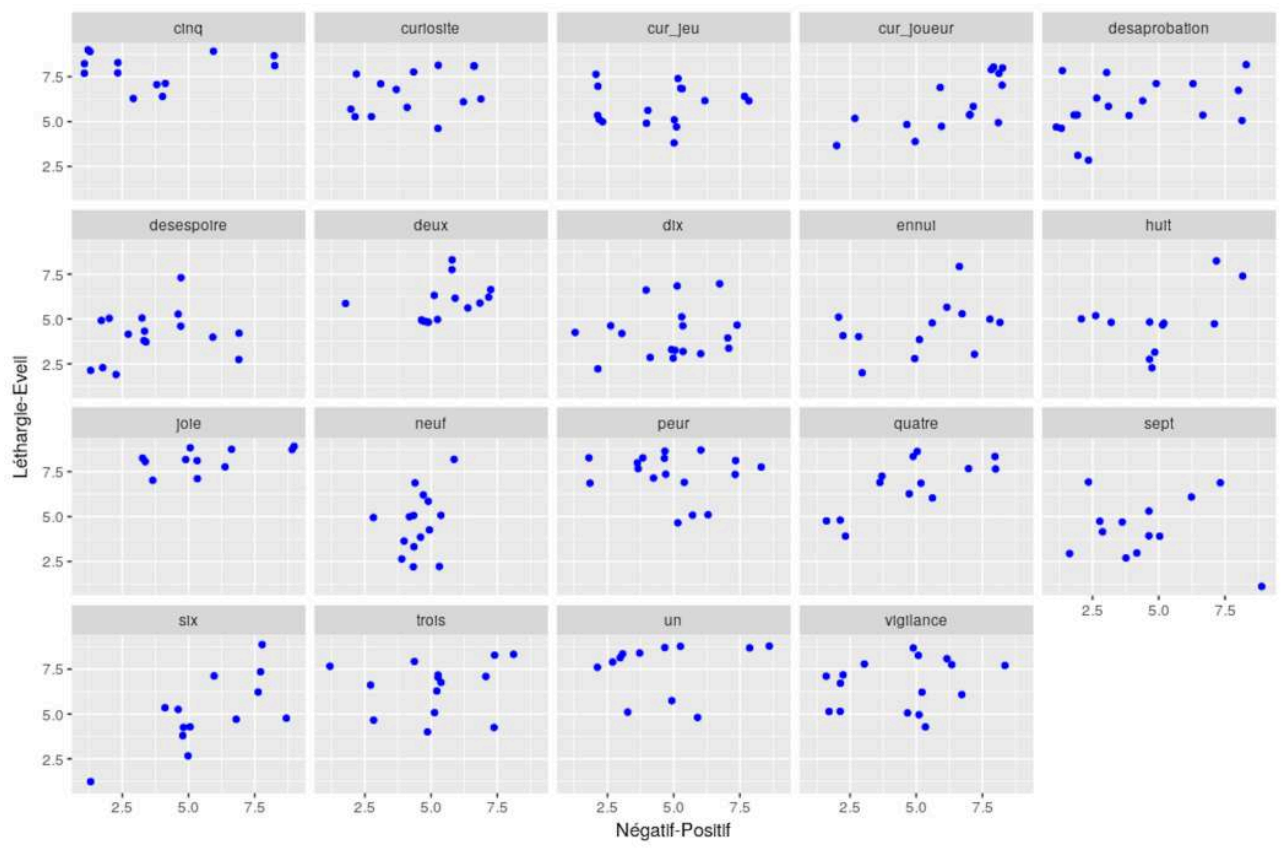

4 : Distribution des valeurs accordées par les participants à chaque mouvement.

\section{Conclusion}

L'expérience présentée ici s'inscrit dans un intérêt plus général pour l'interaction entre humains et machines. Elle contribue à la formalisation des interactions entre humains et machines, en cherchant notamment à mettre au jour leurs propriétés spécifiques et à en appréhender la rationalité propre. Elle s'inscrit en cela dans un travail qui vise à mettre en évidence la façon dont les sujets sont amenés à modifier leurs comportements sous l'influence du comportement de la machine, c'est-à-dire à rendre compte des changements induits par les effets de ce type d'interaction spécifique. En insistant sur les aspects du comportement non verbal, ces travaux sont susceptibles d'apporter des résultats à propos des processus impliqués dans le maintien de l'échange. Ils s'inscrivent ainsi dans la continuité des recherches sur l'élaboration d'une 
théorie formelle de l'interaction. Or en la matière, la robotique dite sociale a mis l'accent sur l'imitation du corps humain afin de faciliter les rapports que nous pourrions entretenir avec les machines lorsqu'elles seront en mesure, techniquement, de traiter la complexité des informations issues du monde réel. On doit se demander toutefois si ce pari de la robotique sociale est réaliste. S'il est si simple de chercher à copier la mécanique des interactions humaines et d'en transférer la complexité inhérente à des machines.

L'expérience QUALCOM est ainsi une invitation à prendre en compte l'idée que, pour les personnes qui ont accepté d'y participer, une machine a priori interactive restera toujours étrangère aux relations qui sont entretenues dans la vie quotidienne, entre pairs. De telles interactions ne peuvent échapper aux principes d'un jeu trouble lors duquel chaque "interactant» est contraint de faire comme si (Becker, 2015). Cette étrangeté, que la robotique explore depuis longtemps (Mori 1970), ne consiste pas pour autant en une seule acceptation ou un seul rejet de la qualité d'« interactant » de l'objet mobile. Au contact de la lampe du groupe PsyPhiNe, les participants semblent mettre en jeu tout un ensemble de préconceptions qui leur servent à décoder l'action qu'ils perçoivent, à lui donner du sens, très souvent en imaginant un contexte différent de celui de l'expérience où ces actes pourraient leur paraître plus facile à interpréter ${ }^{7}$. Situés hors d'un contexte d'interaction du fait de la forme donnée à l'expérience, les mouvements de la lampe invitent ainsi les participants à un exercice particulier de la pensée qui les engage à tisser des analogies avec des choses qu'ils connaissent ou des éléments tirés de leur propre expérience. Malgré la taille réduite de l'échantillon, principale limite à nos yeux d'une telle expérience ${ }^{8}$, l'importance de ces conceptions préalables reste pour l'heure sous-estimée par la robotique sociale à l'endroit où elles sont pourtant déterminantes dans l'étude de la communication (Sperber \& Wilson 1989).

L'autre apport de cette expérience est de déplacer la question de l'anthropomorphisme dans les relations entre humains et robots en soulignant le rôle important qu'occupent les analogies dans l'interaction avec une machine. Alors qu'aujourd'hui il est de coutume de considérer en robotique que l'ontologie imputée aux robots relève d'une sorte de personne dont les qualités sont proches de la personne humaine, l'expérience QUALCOM montre que l'identité imputée à une machine suit en réalité des sentiers bien moins balisés qu'on ne pourrait l'imaginer ; qu'elle suit un processus visant à chercher dans son mouvement des fragments d'actions humaine ou animale, ou encore certaines qualités associées à d'autres objets. Cette symétrie avec les procédés de fragmentation et d'assemblage qui caractérisent généralement la construction des robots dits sociaux mérite qu'on lui accorde une attention particulière.

La " personne » dont on suppose l'existence dans les machines doit dès lors être étudiée de plus près. On se rendra peut-être compte, en portant l'intérêt sur la façon dont les participants des expériences en interaction humain-robot décrivent leur rapport envers les machines, de la complexité qu'elles induisent et des difficultés qu'elles font peser sur une relation que les roboticiens aimeraient pourtant naturelle. En la matière, l'expérience QUALCOM suggère qu'il est peut-être temps pour la robotique dite sociale de repenser les limites de l'imitation de la nature et des liens sociaux, et de plutôt chercher comment la coopération avec les machines peut s'établir sur des bases différentes que celles qui caractérisent les rapports ordinaires entre humains. Elle s'inscrit également dans une démarche visant à mieux saisir la compréhension des 
rapports qu'ont les humains et les non-humains en soulignant l'importance qu'occupent les cadres relationnels qui caractérisent les interactions avec ces derniers et leur rôle déterminant dans les processus d'apprentissage de la nouveauté.

Pour prolonger cette dernière idée, une telle expérience permet de souligner que les créatures artificielles dont nous sommes prêts à nous entourer forment, dans notre esprit, une espèce propre, paradoxalement en voie de constitution, à propos de laquelle nous savons trop peu de choses et dont l'impact sur nos sociétés reste encore difficile à anticiper (Grimaud \& Becker 2019). Notons pour conclure que les rapports qui sont entretenus ailleurs, avec des entités comparables (en cela qu'elle constituent comme les robots des identités aussi complexes que vagues), inscrivent toujours leurs activités dans des canaux particuliers de la communication, qui restent étrangers à ceux généralement employés par les humains, mais pas moins marqués par la culture et les agencements sociaux-matériels (notamment au niveau des médiations) que cette dernière met en œuvre pour rendre cette communication possible.

Barrett, L. F. \& J. A. Russell 1998 « Independence and Bipolarity in the Structure of Current Affect ", Journal of Personnality and Social Psychology 74(4) : 967-984.

Becker, J. 2012a « Le corps humain et ses doubles. Sur les usages de la fiction dans les arts et la robotique », Gradhiva $15: 102-119$

Becker, J. « La mécanisation du lien social », Humain augmenté : état des lieux et perspectives critiques. Colloque de l'Institut des Sciences de la Communication du CNRS, décembre 2012. [En ligne] : https://www.iscc.cnrs.fr/IMG/pdf/

JBecker_Mecanisation.pdf?1033/c003ebe2fd6edb12d1f4865d652289f8a331537d.

Becker, J. 2015 Humanoïdes, expérimentations croisées entre arts et sciences. Nanterre :

Presses Universitaires de Paris Ouest.

Descola, P. 2005 Par-delà nature et culture. Paris : Gallimard.

Espinas, A. 1903 « L'organisation ou la machine vivante en Grèce au IV siècle avant J.-

C. ", Revue de métaphysique et de morale $11: 702-715$

Giard, A. 2017 Un désir d'humain. Les « love doll » au Japon. Paris : Les Belles lettres.

Grimaud, E. \& J. Becker 2019 (à paraitre) « The Kinetics of Suspicion », in S. Bianchini \& E. Quinz dir., Behavioral Objects, vol. 2. Berlin : Sternberg Press.

Grimaud, E. \& Z. Paré 2011 Le jour où les robots mangeront des pommes. Conversations avec un Geminoid. Paris : Petra.

Grimaud, E. \& A.-C. Taylor dir. 2016 Persona, étrangement humain. Paris-Arles : Musée du quai Branly - Actes Sud.

Guthrie, S. 1980 « A Cognitive Theory of Religion », Current Anthropology 21(2) : 181-203.

Heider F. \& M. Simmel 1944 « An Experimental Study of Apparent Behavior », The

American Journal of Psychology 57(2) : 243-259.

Helmreich, S. 1998 Silicon Second Nature. Culturing Artificial Life in a Digital World.

Oakland : University of California Press.

Kohn, E. 2017 Comment pensent les forêts. Paris : Zones sensibles.

Lasseter, J. 2001 " Tricks to Animating Characters With a Computer ", Newsletter, ACM SIGGRAPH Computer Graphics 35(2) : 45-47.

Latour, B. 2010, «Prendre le pli des techniques », Réseaux 5(163) : 11-31.

Mindell, D. A. 2015 Our robots, Ourselves, Robotics and the Myths of Autonomy. New York : Viking.

Mori, M. 1970 « Bukimi no tani (The Uncanny Valley)», Energy 7(4) : 33-35.

Pitrou, P. 2016 « Êtres vivants/artefacts, processus vitaux/processus techniques :

remarques à propos d'un cadran analytique », in P. Pitrou, L. Coupaye \& F. Provost dir. 
Des êtres vivants et des artefacts, Paris (« Les actes ») [En ligne] : actesbranly.revues.org/ 653.

Reeves, B. \& C. Naas 1996 The Media Equation : How People Treat Computers, Television and New Media Like Real People and Places. Cambridge : Cambridge University Press.

Richardson, K. 2016 « Sex Robots Matters : Slavery, the Prostituted, and the Rights of Machines ", IEEE Technology and Society Magazine 35(2) : 46-53.

Riskin, J. 2003 « The Defecating Duck or the Ambiguous Origins of Artificial Life », Critical Inquiry 29(4) : 599-633.

Santos-Granero, F. dir. 2009 The Occult Life of Things, Native Amazonian Theories of Materiality and Personhood. Tucson : University of Arizona Press.

Siciliano B. \& O. Khatib dir. 2016 Handbook of Robotics. Springer International Publishing. Sperber, D. \& D. Wilson 1989 La Pertinence. Communication et cognition. Paris : Les Éditions de Minuit.

Suchman, L. 2007 Human-Machine Reconfiguration, Plans and Situated Actions, $2^{\text {nd }}$ éd. NewYork : Cambridge University Press

Tisseron, S. 2015 Le jour où mon robot m'aimera. Vers l'empathie artificielle. Paris : Albin Michel.

Vidal, D. 2007 « Anthropomorphism or Sub-Anthropomorphism ? An Anthropological Approach to Gods and Robots ", Journal of the Royal Anthropological Institute 13(4) :

917-933

Vidal, D. 2017 Aux frontières de l'humain. Dieux, figures de cire, robots et autres artefacts.

Paris : Alma Éditeur

Viveiros De Castro E. 2014 « Perspectivisme et multinaturalisme en Amérique indigène ", Journal des anthropologues 138-139 : 161-181.

\section{NOTES}

1. Rappelons ici que l'étrangeté suscitée par les robots anthropomorphes fait l'objet de recherches approfondies en robotique depuis les années 1970 (Mori 1970).

2. On voit ici, mais selon une perspective qui n'est pas seulement technicienne, combien l'autonomie d'une machine ne peut être pensée indépendamment du contexte où elle s'exerce (Mindell 2015).

3. http://www.ben-dror.com/pinokio.

4. On doit noter ici que l'emprunt à la forme d'une lampe d'architecte n'évacue pas complètement une référence biomorphique. Le court métrage Luxo Jr constitue en réalité une véritable plateforme expérimentale au sein de laquelle John Lasseter s'est appliqué à donner aux spectateurs les moyens de projeter une intériorité, des buts ou encore des émotions envers les protagonistes du film (Lasseter 2001). Rappelons par ailleurs qu'en matière d'animation, l'absence de référence anthropomorphique n'empêche pas aux projections de ce type de prendre forme chez le spectateur (Heider \& Simmel 1944).

5. Notons que l'animation de la lampe ressemble beaucoup aux principes d'animation par interpolation décrits dans (Becker 2015).

6. Une expérience plus récente montre que l'ampoule située dans la «tête » de la lampe est souvent considérée comme un œil. 
7. Cette façon de reconstruire du sens rejoint dans une certaine mesure l'économie cognitive des processus anthropomorphiques décrite par Stewart Guthrie (1980).

8. Notons toutefois que l'expérience se poursuit en ligne à l'adresse suivante: http:// tangriam.msh-lorraine.fr/.

\section{ABSTRACTS}

Many roboticists are convinced that the humanization of machines will make the communication between humans and robots easier. However, despite their efforts to understand which basic social elements are needed to build a natural relationship with them, social robots always trig uncanny feelings. Taking an experiment in human-robot interaction as an example, we will study how the participants comment the activity of a robotic lamp. This study will show how its movements are interpreted, how its nature is considered and how it is subject to a strong variation during the interaction. Looking closely how participants engage themselves in a difficult exercise of reconstruction of the sense, we will see that such an interaction rely not that much on a particular projection of human attributes on perceived movements but rather on a complex set of analogies.

Beaucoup de roboticiens restent aujourd'hui convaincus que l'humanisation des machines permettra de faciliter la communication entre les humains et les objets qu'ils conçoivent. Or, malgré leurs efforts pour saisir les éléments de base permettant de construire une relation naturelle avec eux, les robots dits sociaux restent toujours marqués par l'étrangeté. Partant d'une expérience, nous étudierons les commentaires suscités par l'activité d'une lampe robotisée. Cette étude permettra de montrer de manière plus claire comment ses mouvements sont interprétés, en quoi consiste sa nature et comment celle-ci est soumise à une forte variation dans le temps même de l'interaction. À travers un exercice difficile de reconstruction du sens auquel les participants de l'expérience se prêtent de bon gré, nous verrons qu'une telle interaction implique pour eux la mise en œuvre non pas d'une pensée qui viendrait projeter sur le mouvement perçu un caractère d'emblée humain mais dépendant plutôt d'un ensemble complexe d'analogies.

\section{INDEX}

Keywords: Anthropomorphism, Animism, Experimentation, Interdisciplinarity, Human-Machine Interaction, Robots

Mots-clés: Anthropomorphisme, Animisme, Expérimentation, Interdisciplinarité, Interaction humain-machine, Robots

\section{AUTHORS}

\section{JOFFREY BECKER}

Docteur en anthropologie sociale et ethnologie et chercheur affilié au Laboratoire

d'anthropologie sociale (LAS Collège de France). Ses recherches portent sur les relations entre 
humains et machines. Elles visent à mieux saisir les dynamiques qui font des machines dites intelligentes des outils expérimentaux, dont l'activité questionne nos modèles sur un plan à la fois ontologique, interactionnel et social.

\section{VIRGINIE ANDRÉ}

Maître de conférences en sciences du langage à Université de Lorraine-CNRS et membre du laboratoire ATILF depuis 2007. Elle est spécialiste de l'analyse sociolinguistique des interactions verbales, dans des situations ordinaires et en situation de travail. Ses travaux se situent également à l'interface entre l'analyse des interactions et l'enseignement/apprentissage sur corpus du français parlé.

\section{ALAIN DUTECH}

Chargé de recherche pour l'INRIA en informatique au Laboratoire lorrain de recherche en informatique et ses applications (LORIA) de Nancy. Il a obtenu une thèse en Intelligence Artificielle en 1999 et une habilitation à diriger les recherches en 2010. Il s'intéresse aux fondements de la cognition, aussi bien artificielle que naturelle. 